\title{
Analysis of digital fences against COVID-19
}

\author{
Yoshiyasu Takefuji ${ }^{1}$
}

Received: 23 August 2021 / Accepted: 14 September 2021 / Published online: 17 September 2021

(c) IUPESM and Springer-Verlag GmbH Germany, part of Springer Nature 2021

\begin{abstract}
This paper examines individual policies against the covid-19. The number of deaths due to COVID-19 can be divided by the population (in million) to score the performance of individual policies. 19 countries were investigated. Although many experts emphasize pharmaceutical methods including vaccines, the most effective policy against the covid-19 is based on a robust digital fence where the physical isolation strategy reveals the remarkable achievement for mitigating the pandemic. To mitigate and end the pandemic, combined policies with vaccination and digital fencing need to be used.
\end{abstract}

Keywords Digital fence $\cdot$ Comparative policy $\cdot$ COVID-19 pandemic $\cdot$ Vaccine $\cdot$ Isolation

\section{Introduction}

Vaccines against COVID-19 are available, but not yet globally and publicly available. COVID-19 virus variants are increasing. Therefore, other ways to control and mitigate the spread of COVID-19 disease, including case identification, isolation, testing, contact tracing, quarantine and location tracking must be found to break the chain of transmission. In this paper, we report what a digital fence is and the impact of individual policies on the COVID-19 pandemic. There are four types of digital fences: no digital fence, incomplete digital fence, leaky digital fence, and robust digital fence. A robust digital fence plays a key role in mitigating pandemics. The number of deaths due to COVID-19 can be divided by the population (in millions) to score the performance of individual policies. 19 countries were studied and three groups emerged: a group with less than 300 score, a group between 700 and 1000, and a group with over 1400 respectively.

In June 2020, the survey report entitled "Regulating electronic means to fight the spread of COVID-19" was published [1]. The report surveys the regulation of electronic means to fight the spread of COVID-19 in 23 selected jurisdictions around the world including Argentina, Aus-

This article is part of the COVID-19 Health Technology: Design, Regulation, Management, Assessment

Yoshiyasu Takefuji

takefuji@keio.jp

1 Faculty of Data Science, Musashino University, 3-3-3 Ariake Koto-ku, Tokyo 135-8181, Japan tralia, Brazil, China, England, France, Iceland, India, Iran, Israel, Italy, Japan, Mexico, Norway, Portugal, the Russian Federation, South Africa, South Korea, Spain, Taiwan, Turkey, the United Arab Emirates (UAE), and the European Union (EU). In this paper, 19 jurisdictions including China, New Zealand, Taiwan, Australia, South Korea, Iceland, Japan, UAE, India, Israel, Russia, Sweden, France, Spain, Mexico, the US, the UK, Italy and Brazil were investigated.

In the majority of the surveyed countries, downloading COVID-19 apps is voluntary [1].

Some of the surveyed countries have also established electronic databases for logging infected individuals and/or contract tracing. South Korea has developed an electronic wristband that monitors people's compliance with selfquarantine; however, it is not mandatory and violators must consent to wearing it $[1,2]$.

Taiwan's "digital fence" monitors the location of those required to undergo home quarantine via their own cellphones or government-issued cellphones, with the goal of preventing their movement [1]. The goal of the digital fence is to isolate asymptomatic [3] and pre-symptomatic [4] carriers from uninfected individuals for mitigating the pandemic. Therefore, infection testing plays a key role in identifying infected individuals.

In the UAE, people who are ordered to quarantine must install an app, which sends alerts to them to stay within the range of movement allowed during the quarantine and provides health authorities with the precise location of these individuals [1]. 
There are four types of a digital fence: no digital fence, an incomplete digital fence, a leaky digital fence, and a robust digital fence. An incomplete digital fence means that the digital fence has not been fully applied to the country. A leaky digital fence means that coronavirus Apps using smartphones are optional or voluntary while a robust digital fence using mandatory coronavirus Apps.

\section{Methods and result}

This paper examines how effective digital fence is for mitigating the pandemic. Digital fence types of 19 selected jurisdictions as of June 2020 [1] and the number of deaths due to COVID-19 as of June 252021 from Worldometers are summarized in Table 1. The last column in Table 1 shows individual scores of 19 jurisdictions where each integer score is calculated by dividing the number of deaths by the population of individual country.

The lower the score, the better the result can be achieved from the viewpoint of human perspectives. Scores can be achieved through individual policies and the culture of citizens.

Figure 1 indicates there are three distinguished groups. One group is a set of countries with less than 300 . The second group is that of countries with score 700 and 1000 . The last group is composed of countries with over 1400 .

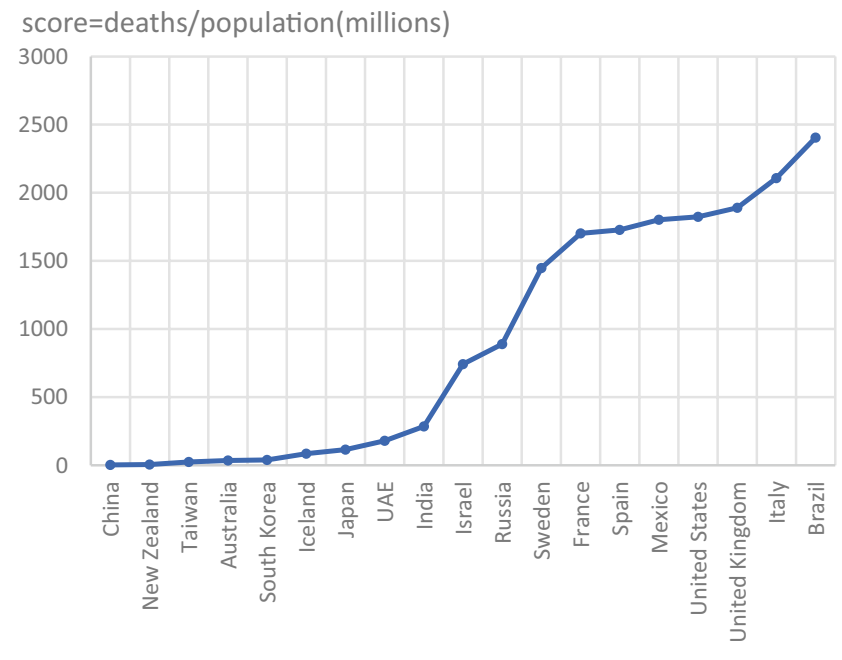

Fig. 1 shows the scores of 19 selected jurisdictions in increasing order. In Fig. $1 \mathrm{X}$-axis indicates the $\mathrm{x}^{\text {th }}$ country while $\mathrm{Y}$-axis shows the score of the $\mathrm{x}^{\text {th }}$ country where $\mathrm{x}$ is a natural number between 1 and 19.

China, New Zealand, Taiwan, Australia, South Korea, Iceland, Japan, UAE and India belong to the first group. Israel and Russia belong to the second group while Sweden, France, Spain, Mexico, the US, the UK, Italy and Brazil belong to the worst score group.

Figure 1 clearly shows that a robust digital fence policy using mandatory Apps can produce a good score for
Table 1 Digital fence types as of June 2020 and no. of deaths as of June 252021

\begin{tabular}{|c|c|c|c|c|}
\hline country & deaths & population & score & Digital fence types \\
\hline China & 4636 & 1439.32 & 3.2 & $\begin{array}{l}\text { unknown but officials saying } \\
\text { robust }\end{array}$ \\
\hline New Zealand & 26 & 4.82 & 5.4 & robust \\
\hline Taiwan & 610 & 23.82 & 25.6 & robust \\
\hline Australia & 910 & 25.5 & 35.7 & Leaky with COVIDSafe \\
\hline South Korea & 2012 & 51.27 & 39.2 & robust \\
\hline Iceland & 29 & 0.34 & 85.3 & leaky \\
\hline Japan & 14582 & 126.48 & 115.3 & no fence \\
\hline UAE & 1782 & 9.89 & 180.2 & semi-robust \\
\hline India & 394493 & 1380 & 285.9 & leaky to robust \\
\hline Israel & 6429 & 8.66 & 742.4 & leaky but robust for tracing \\
\hline Russia & 129869 & 145.93 & 889.9 & leaky to robust \\
\hline Sweden & 14619 & 10.1 & 1447.4 & no fence \\
\hline France & 111101 & 65.27 & 1702.2 & leaky \\
\hline Spain & 80779 & 46.75 & 1727.9 & leaky \\
\hline Mexico & 232346 & 128.93 & 1802.1 & leaky \\
\hline United States & 603527 & 331 & 1823.3 & no fence \\
\hline United Kingdom & 128330 & 67.89 & 1890.3 & leaky \\
\hline Italy & 127418 & 60.46 & 2107.5 & leaky \\
\hline Brazil & 511142 & 212.56 & 2404.7 & incomplete \\
\hline
\end{tabular}


mitigating the pandemic. Taiwan, China, New Zealand, Iceland and South Korea use their robust policy using mandatory Apps. UAE uses a semi-robust policy.

\section{Discussions}

The best score group is composed of Australia, China, Japan, South Korea, Taiwan, Iceland, UAE, and New Zealand.

The worst group did not use the robust digital fence policy. Japan did not use any digital fence at all but it belongs to the best score group. As far as we know, there is no scientific evidence on this mysterious fact on Japan. Culture differences may influence the mitigation where traditional Japanese culture does not include handshakes, hugs, or kisses, but it does include wearing a mask. All of Japanese culture can happen to disturb the spread of infectious diseases [5]. In other words,

We will investigate individual countries of the best score group using a leaky digital fence: Australia, Iceland, and UAE respectively.

Although coronavirus Apps in Australia are voluntary, anonymized location data and mobile data in contact tracing can be used by Australian government for mitigating the pandemic [1]. In Australia, useful data on smartphones can be legally obtained from the carriers of infected individuals [1].

If a user in Iceland is later confirmed infected with COVID-19, the Icelandic Contact Tracing Team requests access to the location data from the phone [1]. While the app has been downloaded by $40 \%$ of the population, it has mainly served to complement ordinary contact tracing methods. "Rakning C-19" App is voluntary in Iceland. Selfquarantining is less restrictive than isolation, but persons must follow the guidelines. Under Icelandic law, persons who have COVID-19 must isolate for the duration of their illness, meaning they must stay at home alone or together with a person who is also isolating. Violating quarantine and isolation rules is punishable by monetary fines.

Infected individuals in UAE will be asked to download a mobile app "TraceCovid" [1]. The app ensures that the quarantined person adheres to mandatory requirements. The main purpose of the app is to send alerts that inform users to stay within the range of movement allowed during the quarantine. The app provides the health authorities with the precise location of these individuals to ensure that they do not violate the quarantine.

Leaky digital fences or incomplete digital fences can produce severe scores except Japan.

Taiwan's digital fence policy in the beginning was the best among 19 jurisdictions. However, Taiwan, once a
COVID-19 success story is now struggling with testing backlog amid largest outbreak. People suspected of having Covid-19 are waiting up to nine days for a confirmed result in Taiwan [6]. Crucially, on April 15, Taiwan relaxed its quarantine rules for unvaccinated pilots and aircrew [7]. An outbreak of COVID-19 was triggered in Taiwan when unvaccinated pilots and aircrew underwent relaxed quarantine [7]. Swift COVID-19 testing plays an important role in contact tracing.

Israel is the first country to be vaccinated with the highest vaccination rate in the world. Vaccines alone did not end the covid-19 pandemic. Initially, vaccines were effective against COVID-19 in Israel. However, due to the recent SARS-CoV-2 variants, spike mutations, and immune escape [8], Israel is currently experiencing a strong resurgence in daily deaths. The digital fence and improved vaccines against SARS-CoV-2 variants play a key role in mitigating the pandemic.

\section{Conclusion}

The consequence of individual coronavirus policies reveals that a robust digital fence using the state-of-the-art technology is one of the most effective method regardless of vaccine against the covid-19. We need to investigate why calculated scores (the number of deaths / population) can split 19 jurisdictions into 3 groups. We need to investigate why Japan with no digital fence belongs to the best score group. Evidence-based data showed that a robust policy using mandatory coronavirus Apps plays a key role in successfully mitigating the pandemic. Taiwan's digital fence policy in the beginning was the best among 19 jurisdictions. However, Taiwan's result showed that swift COVID-19 testing plays an important role in contact tracing in digital fence.

Israel's result shows that vaccines alone cannot end the covid-19 pandemic. Therefore, digital fence and improved vaccination are significant for ending the pandemic.

Funding No fund.

\section{Declarations}

Informed consent NA

Research involving human participants and/or animals NA

Conflicts of interest The author has no conflict of interest. 


\section{References}

1. Law library of congress. Regulating Electronic Means to Fight the Spread of COVID-19. 2020. https://tile.loc.gov/storageservices/service/11/llglrd/2020714995/2020714995.pdf.

2. Park Han-na. Tracking wristband launched to deter quarantine breakers. 2020. http://www.koreaherald.com/view.php?ud= 20200427000967.

3. Sayampanathan AA et al. Infectivity of asymptomatic versus symptomatic COVID-19. The Lancet. 2021;397(10269):93-94.

4. Julia Ries. Most COVID-19 Cases Come from People Without Symptoms . 2021. https://www.healthline.com/health-news/mostcovid-19-cases-come-from-people-without-symptoms.

5. Iwasaki Akiko et al. Why does Japan have so few cases of COVID19? EMBO Molecular Medicine. 2021;12:e12481.
6. Samson Ellis. Nine-Day Wait for Covid Test Results Leaves Taiwan in Limbo. 2021. https://www.bloomberg.com/news/ articles/2021-05-26/backlogged-test-facilities-leave-taiwanrevising-covid-totals.

7. Ron Hanson. Taiwan's Covid response was once the envy of the world. What went wrong?. 2021. https://thespinoff. co.nz/socie ty/02-06-2021/taiwans-covid-response-wasonce-the-envy-of-the-world-what-went-wrong/.

8. Harvey WT, Carabelli AM, Jackson B et al. SARS-CoV-2 variants, spike mutations and immune escape. Nat Rev Microbiol. 2021;19:409-424. https://doi.org/10.1038/ s41579-021-00573-0.

Publisher's Note Springer Nature remains neutral with regard to jurisdictional claims in published maps and institutional affiliations. 